\title{
Pectus excavatum and pectus carinatum in Dogs
}

\author{
Claudia Luvian de Souza', Giovanna de Oliveira Baia'2, Bianca Costa Rezende', \\ Lorena Tavares de Brito Nery Jaworski', Daniela Prass', Pedro Eduardo Brandini Néspoli³, \\ Arleana do Bom Parto Ferreira de Almeida ${ }^{3}$ \& Valéria Régia Franco Sousa ${ }^{3}$
}

\begin{abstract}
Background: Deformities of the anterior thoracic wall are called pectus: pectus excavatum and pectus carinatum. Pectus excavatum is characterised by dorsal deviation of the caudal region of the sternum while pectus carinatum consists of protrusion of the sternum and/or adjacent cartilage. Both defects may remain symptom-free, but respiratory and cardiac abnormalities have been reported. Another deformity observed in dogs is the swimming dog syndrome, which consists of the lateral opening of the thoracic and pelvic limbs, associated with the pedalling movement. These disorders are frequently reported in medical practice, are considered rare among canines. This report aims to describe a case of pectus excavatum associated with the swimming dog syndrome (Case 1) and another case of pectus carinatum (Case 2).

Cases: Case 1. An approximately 45-day-old male American Pitbull canine had difficulty standing. Physical examination revealed hyperextension of the thoracic and pelvic limb joints and flattening of the thorax. After radiographic examination, pectus excavatum and the swimming dog syndrome were confirmed. The conservative treatment with splinting and hydrotherapy was chosen. At the 3-month follow-up, slight improvement in the limbs and irregular and unsatisfactory growth of the thorax leading to episodes of dyspnoea were observed. Case 2. A female Pug, approximately 2 months old, had a thorax deformity. Physical examination revealed thorax protrusion, confirmed on radiography as pectus carinatum. Conservative therapy was provided using compressive bandage. One month later, the tutor reported improvement in the condition and absence of respiratory changes.

Discussion: Pectus deformities has low incidence, and cases to pectus carinatum, there is a sexual predisposition, that is, males are more predisposed, differing from Case 2, a female dog. In the cases of pectus excavatum, no genetic alteration was directly related to its occurrence, although familial occurrence is reported in humans, as in Case 1, in which the patient was the only one of this litter to present this deformity. Though the possibility is lower, the acquired aetiology has been described in other cases. In pectus carinatum, there is the possibility of an acquired aetiology, which corroborates Case 2, considering that the canine had no history of inbreeding, nor family history. However, it is important consider racial predisposition, where there is an increasing number of cases of pectus carinatum in brachycephalic canines. Respiratory symptoms are frequently reported in cases of pectus excavatum, which the evolution of the condition occurred in Case 1 , that can be explained by the displacement of organs or the restriction to ventilation. Humans have shown that pectus excavatum reduces static lung function. Case 2 showed no clinical signs, despite this presented dextrocardia, which in the future may confer cardiac dysfunctions. The diagnosis for pectus is considered simple. Performed only by radiography, it can be complemented by echocardiography. In both cases, conservative treatment was the choice. However, in Case 1 there was no success due to the degree of patient involvement, unlike Case 2, which had a better development of the thoracic wall, ensuring better quality of life for the patient. Thus, it is possible to conclude that some differences were found in these two reported cases when compared to others described in the literature. The radiographic diagnosis is confirmatory, and the therapy must be adequate to the degree of thoracic wall deformity. In addition, the prognosis is more reserved for pectus excavatum than pectus carinatum.
\end{abstract}

Keywords: thoracic deformity, dyspnoea, pigeon breast, funnel chest. 


\section{INTRODUCTION}

Anterior thoracic wall deformities, universally designated as pectus, are frequently observed in the medical practice [3]; and can be presented in two types: pectus excavatum and pectus carinatum. Pectus excavatum is characterised by dorsal deviation of the caudal region of the sternum and associated cartilages or narrowing of the caudal region of the entire thorax in which the ribs and sternum grow abnormally, also known as the funnel chest [13]. Pectus carinatum is characterised by a protrusion of the sternum and/ or adjacent costal cartilages, also known as pigeon's breast [11]. It is a congenital disorder sporadically reported in the thoracic wall. Both defects can remain symptom-free, although pectus excavatum can lead to severe dyspnea and abnormalities in cardiovascular function, while pectus carinatum can cause transient respiratory distress [7]. Another deformity observed in neonatal dogs is the swimming dog syndrome in which the thoracic and pelvic limbs are laterally arranged [16].

The swimming dog syndrome and pectus excavatum can occur independently or concomitantly, and its main clinical signs are the inability to remain stationary or walking, with laterally open limbs and movements performed only laterally, such as "pedalling" [6]. Though, pectus is frequently reported in human, for dogs these deformities are rare. And pectus excavatum, when compared to pectus carinatum has an even lower incidence that has been a limiting factor in therapy [9]. Therefore, the aim of this article is to report the progression of pectus excavatum associated with the swimming dog syndrome and pectus carinatum in dogs.

\section{CASES}

Case 1. An approximately 45-day-old male American Pitbull canine, weighing $4.2 \mathrm{~kg}$ (Figure $1 \mathrm{~A})$, was presented to the Veterinary Hospital with difficulty in staying in season. In the general physical examination, all parameters (heart rate $116 \mathrm{bpm}$, respiratory rate $40 \mathrm{bpm}$, and temperature $38.4^{\circ} \mathrm{C}$ ) were within the normal limits, with hyperextension of the thoracic and pelvic limbs and flattening of the thorax. For diagnostic confirmation, thoracic and limbal radiographic examinations were requested. Laterolateral and ventrodorsal radiographs revealed lordosis of the thoracic spine between the $\mathrm{T} 7$ and $\mathrm{T} 10$ vertebrae in the midthoracic position and scoliosis, with lateral deviation to the left at T10. In addition, there was flattening and uniform displacement of the dorsal sternum, with cardiac silhouette deviated dorsally and laterally to the left (Figure 1 B \& C). Radiographic findings of the sternum were compatible with pectus excavatum, and the thoracic limbs suggested the swimming dog syndrome (Figure 1 D). Additionally, echocardiography and haematological examination were performed, with values within the normal range for the species and age.

As therapy, splints were placed approaching the limbs, and hydrotherapy was recommended. Furthermore, the support of analgesia and chondroprotection was provided. The tutor returned with the canine after 5 days, reporting slight improvement with slight flexion of the right hind limb.

Growth in the thoracic circumference was monitored 1, 2, and 3 months after the first visit. At the first visit, the thoracic circumference was found to follow the growth of the animal. At the 1-month follow-up, the thoracic circumference was $38 \mathrm{~cm}$ cranially and $44 \mathrm{~cm}$ caudally, and the animal weighed $6.6 \mathrm{~kg}$, heart rate 148 bpm, respiratory rate $38 \mathrm{bpm}$, and temperature $39^{\circ} \mathrm{C}$. At the 2-month follow-up, the thoracic circumference increased to $43 \mathrm{~cm}$ cranially and $50 \mathrm{~cm}$ caudally, and the animal weighed $8.4 \mathrm{~kg}$, heart rate $144 \mathrm{bpm}$, respiratory rate $48 \mathrm{bpm}$, and temperature $38.9^{\circ} \mathrm{C}$. At the 3-month follow-up, the growth had stabilised, with the thoracic circumference being $43 \mathrm{~cm}$ cranially and $50 \mathrm{~cm}$ caudally, and the animal weighed $9.7 \mathrm{~kg}$, heart rate $120 \mathrm{bpm}$, temperature $39^{\circ} \mathrm{C}$ and presented with episodes of dyspnoea.

Case 2. An approximately 2-month-old female Pug weighing $1.6 \mathrm{~kg}$ was evaluated for major signs of thorax deformity. In the general physical examination, all parameters were within the normal ranges, and there was apparent protrusion of the thoracic cavity in the ventral sternal region (Figure $2 \mathrm{~A}$ ). The right lateral and ventrodorsal radiographs showed an increase in the thoracic cavity depth, with ventral prominence of the sternum in relation to the abdominal contour, associated with the displacement of the cardiac axis to the right in the ventrodorsal projection (Figure $2 \mathrm{~B}$ ). Radiographic findings confirmed pectus carinatum and dextrocardia, and echocardiography was recommended for better diagnostic elucidation.

Compression bandage therapy was provided, with good acceptance by the animal, without signs 


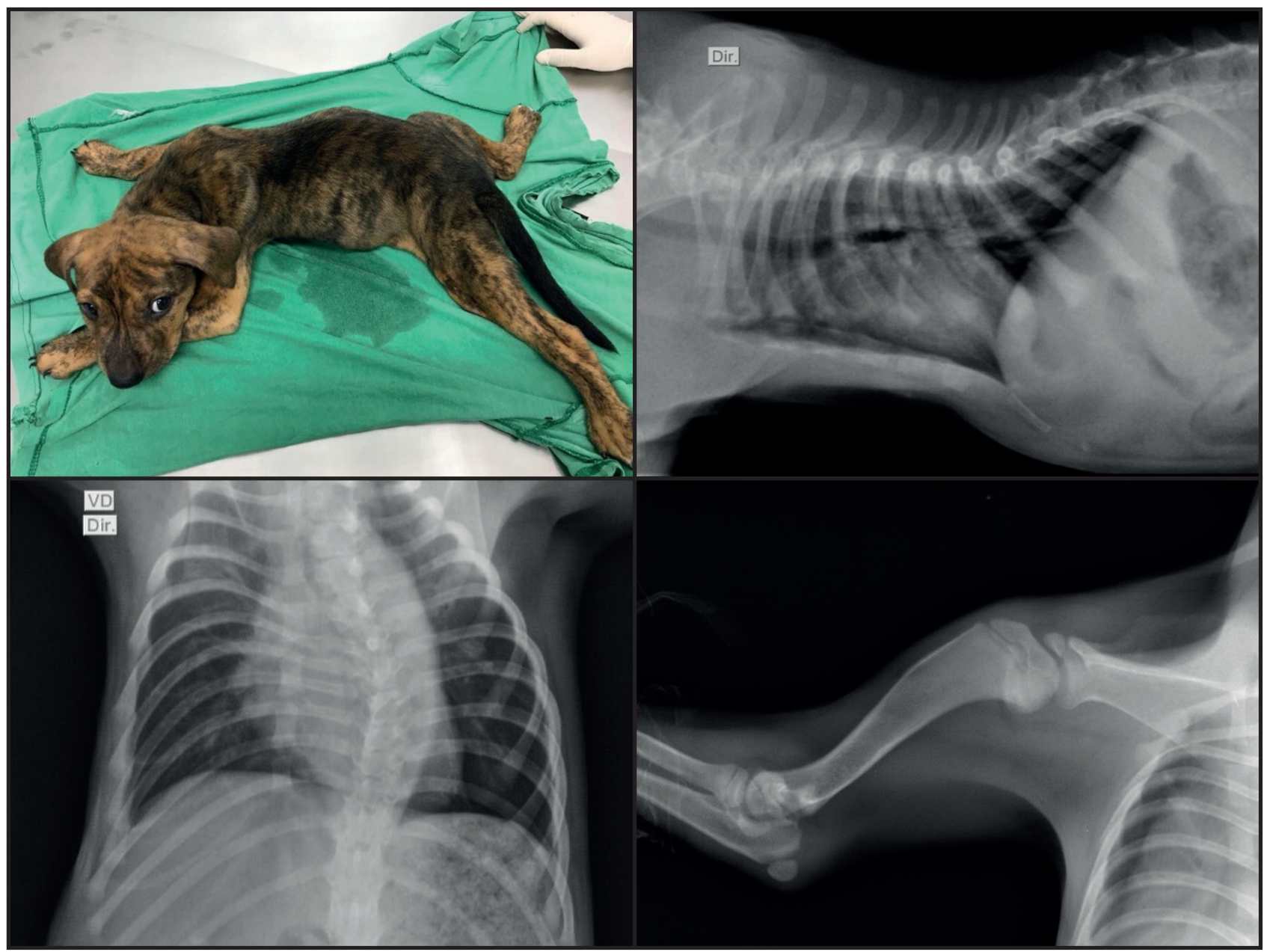

Figure 1. Case 1. A- At the first appointment, the patient shows thoracic flattening and lateralisation of the thoracic and pelvic limbs. B- The thoracic X-ray scan at the right laterolateral projection demonstrates thoracic lordosis, flattening, and dorsal displacement of the sternum. C- Ventrodorsal radiography shows scoliosis in the thoracic portion of the vertebral column $(\mathrm{C})$ at 7 months of age. D- Radiography of the left thoracic limb shows lateralisation of the joints.

of dyspnoea. The tutors were instructed to apply the splint twice a day for 30 days and attend follow-ups for the evolution of the condition in addition to echocardiography examinations, but they did not comply. One month after the first consultation, the patient grew, and her chest followed the growth, and no episode of respiratory alteration was found.

\section{DISCUSSION}

Pectus deformities have a low incidence; however, there is an important sexual predisposition, males generally have a higher incidence of pectus carinatum compared to females [1], although the dog in the present case was a female.

No genetic alterations were directly associated with the occurrence of pectus excavatum, although familial occurrence of the anomaly has been reported in humans. Castration of the affected animals is recommended, since the deformity is usually congenital [10]. In another case, the animal in question was a first-born pup with no history of consanguinity between the parents, and the other litter pup showed no changes. Although this possibility is remote, it is important to note that there is also evidence of animals presenting the acquired shape [8].

The aetiology of pectus carinatum has not been identified, but its occurrence is believed to be acquired [1]. Similarly, in case 1, the Pug had no history of inbreeding between the parents or similar changes in the other litter pups. However, there is also the possibility of racial predisposition, with an increased risk of pectus carinatum in brachycephalic dogs with a screw tail, with Pugs being among the most at risk breeds [7].

Respiratory distress is a clinical sign often reported by the tutor in cases of pectus excavatum, and although this was not the chief complaint at the first 


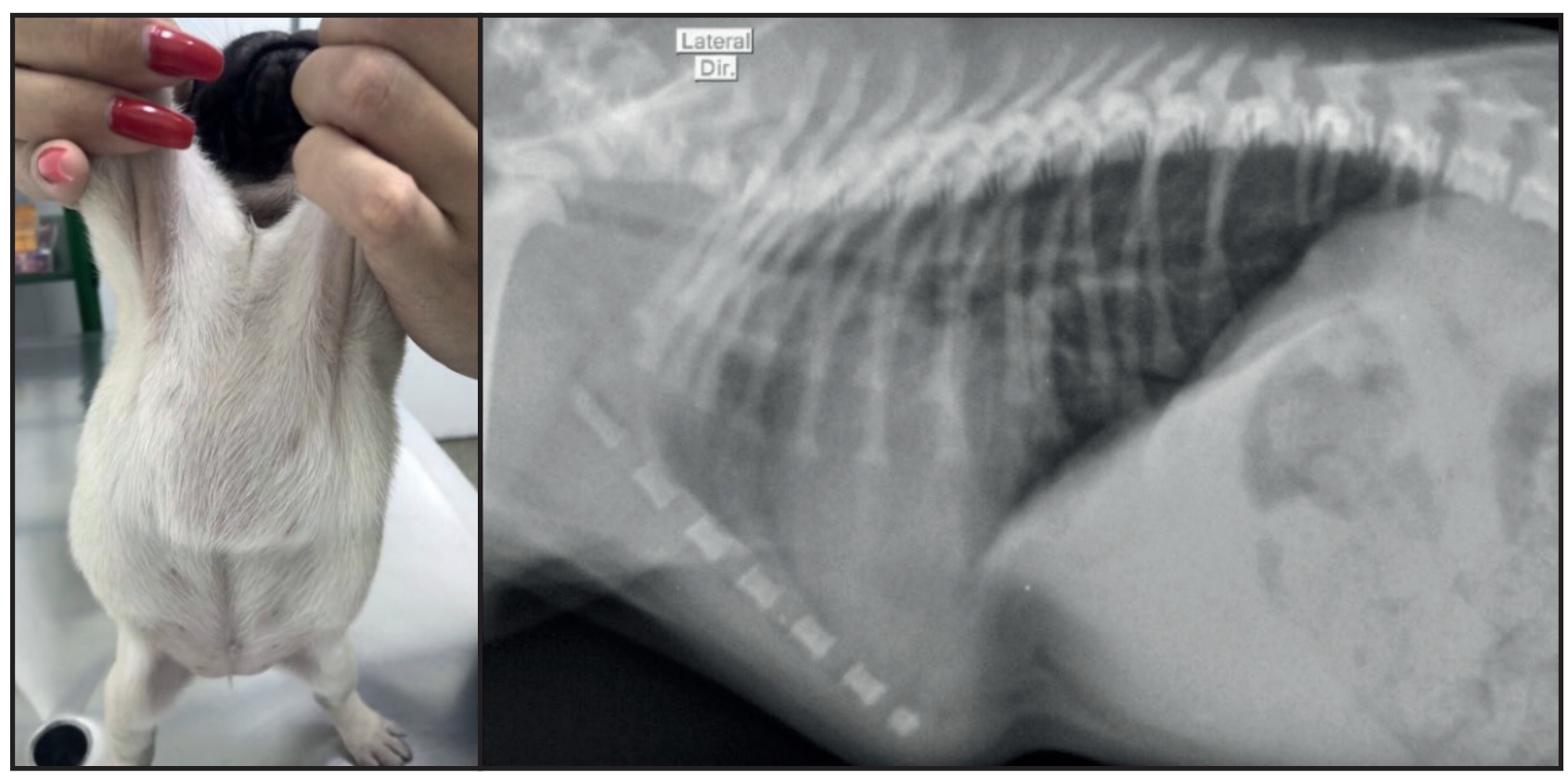

Figure 2. Case 2. A- At the first visit, the patient demonstrates thoracic protrusion. B- The right laterolateral thoracic radiograph shows external caudal angulation in relation to the abdomen and deep thoracic conformation.

consultation, it developed with the growth of the animal, suggesting an association. Displacement of organs or restriction of ventilation and deformity become more pronounced with growth [10]. Likewise, recent studies carried out in humans demonstrate that patients with pectus excavatum have a reduction in static pulmonary function, presenting exercise intolerance, easy fatigue and effortless speech [5].

Like the case reported by Martins et al. [9], in case 2 , no respiratory complications were observed initially or during growth. In contrast, in the case reported by Souza et al. [14], easy tiredness and difficulty breathing were found while performing exercises, and in the case reported by Sanches [12], dyspnoea was found, although without the confirmed occurrence of pectus carinatum. Additionally, case 2 also presented with dextrocardia, which could not be classified into situs inversus, i.e., complete inversion of all the thoracic and abdominal organs, or situs solitus, which is the orientation of normal abdominal organs. Cases of situs solitus associated with dextrocardia may commonly lead to the development of heart failure [15], which may occur throughout the patient's life.

Pectus is simple to diagnose as it is based on the findings of physical examination and imaging [3]. It is possible to analyse the severity the thoracic wall deformity by performing the front-sagittal index in pectus excavatum [4]. In such cases, associated complementary examinations, such as echocardiography, are necessary to assess cardiac positioning, particularly if there are other associated abnormalities [13].

The treatment options for pectus excavatum are conservative treatment, with the use of splint, when there is slight deformation, and partial sternectomy [2]. In the present case, considering that the chances of success would be limited because of the age of the animal and its rigid musculature and bone formation, only the conservative treatment was provided.

In cases of pectus carinatum, conservative measures without surgical intervention are recommended during the growth phase. In dogs that do not show related clinical signs, treatments may not be provided [9]. The use of a compressive splint has adverse effects, such as cyanosis and dyspnoea, which necessitate its immediate removal [14]. Therefore, it is important to assess the needs and risks to the animal. In case 2, the 1-month compressive bandage yielded a good response, with bone growth and no signs of respiratory change.

In view of all the facts presented, it is possible to conclude that some differences were found in these two reported cases when compared to others described in the literature, such as the fact that pectus carinatum occurring in a female. However, the forms of diagnosis already described helped to elucidate these cases, which similarly to those described, thorax X-rays were performed, confirming pectus deformities, and when possible, echocardiography for better diagnostic 
clarification. The prognosis, during the follow-up of the cases, proved to be reserved for pectus excavatum, as case 1 presented severe disease progression regardless of the adopted therapy, while case 2 of pectus carinatum did not show additional clinical signs.
Acknowledgements. Claudia Luvian de Souza thanks for the Comissão Nacional de Residência Multiprofissional em Saúde (CNRMS) fellowship.

Declaration of interest. The authors report no conflicts of interest. The authors alone are responsible for the content and writing of the paper.

\section{REFERENCES}

1 Coelho M.S. \& Guimarães P.S.F. 2007. Pectus carinatum. Jornal Brasileiro de Pneumologia. 33(4): 463-474.

2 Gifford A.T. \& Flanders F.A. 2010. External splinting for treatment of pectus excavatum in a dog with right ventricular outflow obstruction. Journal of Veterinary Cardiology. (12): 53-57.

3 Haje S.A. \& Haje D.P. 2009. Orthopedic approach to pectus deformities: 32 years of studies. Revista Brasileira de Ortopedia. 44(3): 191-198.

4 Hassan E.A., Hassan M.H. \& Torad F.A. 2016. Correlation between clinical severity and type and degree of pectus excavatum in twelve brachycephalic dogs. The Journal of Veterinary Medical Science. 80(5): 766-771.

5 Kelly Junior R.E., Obermeyer R.J. \& Nuss D. 2016. Diminished pulmonary function in pectus excavatum: from denying the problem to finding the mechanism. Annals of Cardiothoracic Surgery. 5(5): 466-475.

6 Kim S.A., Na K.J., Cho J.K. \& Shin N.S. 2013. Home-care treatment of swimmer syndrome in a miniature schnauzer dog. The Canadian Veterinary Journal. 54(9): 869-872.

7 Komsta R., Osinski Z., Debiak P., Twardowski P. \& Lisiak B. 2019. Prevalence of pectus excavatum (PE), pectus carinatum (PC), tracheal hypoplasia, thoracic spine deformities and lateral heart displacement in thoracic radiographs of screw-tailed brachycephalic dogs. Plos One. 14(10): 1-14.

8 Kurosawa T.A., Ruth J.D., Steurer J., Austin B. \& Heng H.G. 2011. Imaging Diagnosis - Acquired pectus excavatum secondary to laryngeal paralysis in a dog. Veterinary Radiology \& Ultrasound. 53(3): 329-332.

9 Martins D.B., Rietjens L.H. \& Petrochi D. 2014. Pectus carinatum em um cão. Acta Scientiae Veterinariae. 42(1): $1-4$.

10 Rahal S.C., Morishin Filho M.M., Hatschbach E., Machado V.M.V., Aptekmann K.P. \& Corrêa T.P. 2008. Pectus excavatum in two littermate dogs. The Canadian Veterinary Journal. 49(9): 880-884.

11 Robicsek F. \& Watts L.T. 2010. Pectus Carinatum. Thoracic Surgery Clinics. 20(2): 563-574.

12 Sanches M.P., Carvalho S.M.R., Costa C.C., Abreu D.M., Nascimento D.M., Jesus W.J., Pessoa G.T. \& Araújo J.R. 2019. Pectus carinatum in a cat. Acta Scientiae Veterinariae. 47(438): 1-4.

13 Singh M. Parrah J.D., Moulvi B.A., Athar H., Kalim M.O. \& Dedmari F.H. 2013. A review on Pectus excavatum in canines: A congenital anomaly. Iranian Journal of Veterinary Sugery. 8(1):59-63.

14 Souza D.B., Andrade Junior P.S.C., Marino C.M.A., Costa F.S. \& Abílio E.J. 2009. Pectus carinatum in a dog. Arquivo Brasileiro de Medicina Veterinária e Zootecnia. 61(1): 276-279.

15 Sparrow D.B., Faqeih E.A., Sallout B., Alswaid A., Ababneh F., Al-Sayed M., Rukban H., Eyaid W.M., Kageyama R., Ellard S., Turnpenny P.D. \& Dunwoodie S.L. 2013. Mutation of HES7 in a large extended family with spondylocostal dysostosis and dextrocardia with situs inversus. American Journal of Medical Genetics. 161(19): 2244-2249.

16 Verhoeven G., De-Rooster H., Risselada M., Wiemer P., Scheire L. \& Bree H. V. 2006. Swimmer syndrome in a Devon rex kitten and na english bulldog puppy. Journal of Small Animal Practice. 47(10): 615-619. 